\title{
Model Atmospheres and Spectra: The Role of Dust
}

\author{
France Allard \\ CRAL-ENS, 46 Allée d'Italie, 69364 Lyon Cedex 07, France \\ Tristan Guillot
}

Observatoire de la Côte d'Azur, BP 4229, 06304 Nice Cedex 04, France

Hans-Günter Ludwig

Lund Observatory, Box 43, 22100 Lund, Sweden

Peter H. Hauschildt and Andreas Schweitzer

Hamburger Sternwarte, Gojenbergsweg 112, 21029 Hamburg, Germany

\author{
David R. Alexander and Jason W. Ferguson \\ Dept. of Physics, Wichita State University, Wichita, KS 67260-0032, \\ USA
}

\begin{abstract}
Brown dwarf atmospheres form molecules, then high temperature condensates (corundum, titanates, silicates, and iron compounds), and then low temperature condensates (ices) as they cool down over time. These produce large opacities which govern entirely their spectral energy distribution. Just as it is important to know molecular opacities ( $\mathrm{TiO}, \mathrm{H}_{2} \mathrm{O}, \mathrm{CH}_{4}$, etc.) with accuracy, it is imperative to understand the interplay of processes (e.g. condensation, sedimentation, coagulation, convection) that determines the radial and size distribution of grains. Limiting case models have shown that young, hot brown (L) dwarfs form dust mostly in equilibrium, while at much cooler stages (late $\mathrm{T}$ dwarfs) all high temperature condensates have sedimented out of their photospheres. But this process is gradual and all intermediate classes of brown dwarfs can partly be understood in terms of partial sedimentation of dust. With new models accounting for these processes, we describe the effects they may have upon brown dwarf spectral properties.
\end{abstract}

\section{Introduction}

Dust forms in the atmospheres of brown dwarfs and late-type $M$ dwarfs. But the radial and surface distributions of the dust are the largest current uncertainties in modelling these objects' spectral and thermal properties. The observation of planetary atmospheres suggests that clouds form in confined layers around the supersaturation level of dust grains. Recent non-grey models addressing 
the issue of dust formation in brown dwarf atmospheres by Tsuji et al. (1996) and Allard et al. (2001) treated dust in pure Chemical Equilibrium (hereafter $\mathrm{CE}$ ). In $\mathrm{CE}$, dust that forms at a given temperature also forms at any lower temperatures. This means that grains were present at all layers above the layer of dust formation onset $(\approx 1800 \mathrm{~K})$, and such CE models represent an extreme or limiting case in the study of dust formation effects in brown dwarfs atmospheres. The confrontation to the observed spectral properties of brown dwarfs could lead to two important conclusions: 1) CE models appear to reproduce the near-IR spectral properties of brown dwarfs (Leggett et al 2000, and references therein), indicating that dust in CE numbers is supported (against gravity) at depths in the atmospheres where the near-IR spectrum of brown dwarfs is emitted;2) CE models could not reproduce the optical and atomic line width properties of brown dwarfs unless dust opacities are ignored in the models' construction (see for example Schweitzer et al. 2000), indicating that dust does not levitate in the uppermost atmospheric layers where these features are formed. Clearly, $\mathrm{CE}$ models are insufficient and clouds are in confined layers in brown dwarfs.

To address this issue, we computed new models including condensation, coagulation and sedimentation in a diffusion approximation based upon Rossow (1978), and an automatic depletion of refractory elements as a result of sedimentation. We also include convective upwelling based on recent three-dimensional radiative hydrodynamical convection simulation results for $\mathrm{M}$ dwarfs by Ludwig, Allard, \& Hauschildt (2002, hereafter LAH02). The models' general premisses and the convective upwelling treatment are detailed below.

\section{Models}

We use the model atmosphere code PHOENIX (version 13) where important opacities and CE calculations are treated as in Allard et al. (2001). The "AMESSettl" models presented here are plane-parallel transfer models where the convection is treated according to the Mixing Length Technique (MLT) inside the convectively unstable (according to the Schwarzchildt criterion) region. The opacity contribution of the strongest atomic lines (Voigt profiles) are accounted for up to $5000 \AA$ from the line center. The model atmospheres are characterized by the following parameters: (i) the surface gravity, $\log (g)$, (ii) the effective temperature, $\mathrm{T}_{\text {eff }}$, (iii) the mixing length to scale height ratio, $\alpha$, here taken to be 2.0 as shown to be the case for M dwarfs by LAH02, (iv) the micro-turbulent velocity $\xi$, here set to $2 \mathrm{~km} / \mathrm{sec}$, and (v) the initial element abundances set here to solar values. For this paper we have calculated a uniform grid of AMES-Settl models ranging from $\mathrm{T}_{\text {eff }}=3000$ to $1000 \mathrm{~K}$ in $100 \mathrm{~K}$ steps, and with gravities ranging from $\log g=3.5$ to 5.5 in steps of 0.5 dex at solar metallicity.

\subsection{The Cloud Model}

At each $\left[\mathrm{T}, \mathrm{P}_{g}\right]$-point of the atmosphere, the CE is solved as described in Allard et al. (2001). For this work we have added to the list of species already included in previous work: some species playing a key role in the formation of alkali atomic lines have been added $(\mathrm{CsCl}, \mathrm{CsCl} / \mathrm{c}, \mathrm{RbCl}, \mathrm{RbCl} / \mathrm{l}, \mathrm{RbCl} / \mathrm{c}, \mathrm{NaCl}$ and $\mathrm{NaCl} / \mathrm{c}$, where /c refers to the crystal form as opposed to the gaseous form, and $/ 1$ refers to liquids). Immediately following this $\mathrm{CE}$ calculation, a diffusion model 

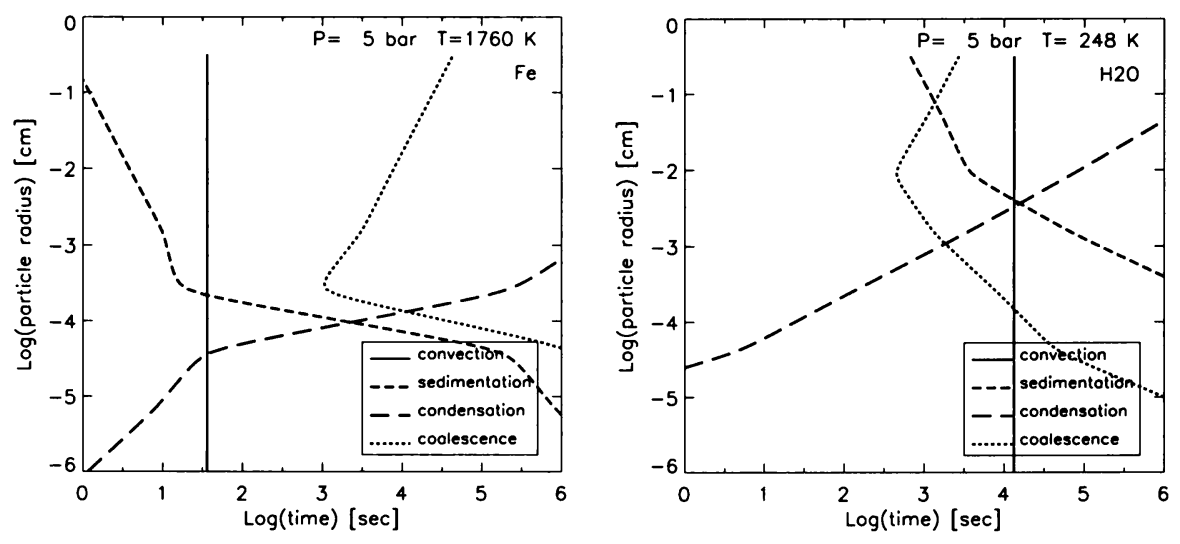

Figure 1. Condensation (1), sedimentation (2), coalescence (3) and convective (4) characteristic time scales as a function of mean particle size. Left panel: ice cloud base assuming a solar abundance of water in the case of a brown dwarf with $\mathrm{T}_{\text {eff }}=1280 \mathrm{~K} ; g=3 \times 10^{5} \mathrm{~cm} / \mathrm{s}^{-2}$. Right panel: Iron cloud base assuming solar abundances in the case of a Jupiter-like planet such that $\mathrm{T}_{\text {eff }}=180 \mathrm{~K}$ and $g=3 \times 10^{3} \mathrm{~cm} / \mathrm{s}^{-2}$.

is applied where the microphysical and convective characteristic time scales are compared (see for example Figure 1). This comparison yields a revised total number density and size of the grains. For the cloud model we assume that grains are heterogeneous i.e. combine all types of condensate in a given layer, but we neglect supersaturation for now. The elements involved in these sedimented grains are then substracted from the initial element abundances, and the CE is solved again to adjust accordingly all gaseous and remaining dust species. We then iterate until the fraction of sedimented grains no longer changes.

As the brown dwarf cools over time, more condensates form and sediment out of the upper atmosphere, leaving the uppermost layers bare of refractory elements. To account for the thermal history of the gas, the cloud model is therefore computed from the innermost, hottest solar abundance layer of the atmosphere to the topmost, coolest one, and the current layer uses the final abundances of the previous layer used as initial values. We use the equations for condensation, coagulation and sedimentation time scales from a planetary cloud microphysic study by Rossow (1978). For the convective upwelling, we compute a "mixing timescale" according to $t_{\text {mix }}=\alpha H_{\mathrm{p}} / v_{\text {conv }}$ where $\alpha$ is the MLT mixing length parameter, $H_{\mathrm{p}}$ is the local pressure scale height, and $v_{\text {conv }}$ the convective velocity where $v_{\text {conv }}$ is set to its MLT value inside the convective zone. Convective mixing causes gas to diffuse upwards in a time scale that increases as a function of depth in the atmosphere. This is the result of a study by LAH02 for a low mass dwarf (see Figure 2a). Inside the radiative zone therefore, we adopt a similar increase which corresponds to a parabolic formula fitted to the MLT values on one side, i.e. with a focus and opening defined by the outermost and innermost convection zone values. Even though Figure $2 \mathrm{ab}$ represent atmospheres that differ by $1000 \mathrm{~K}$, one can appreciate how well our adopted mixing timescale model (full line in Figure 2b) corresponds 

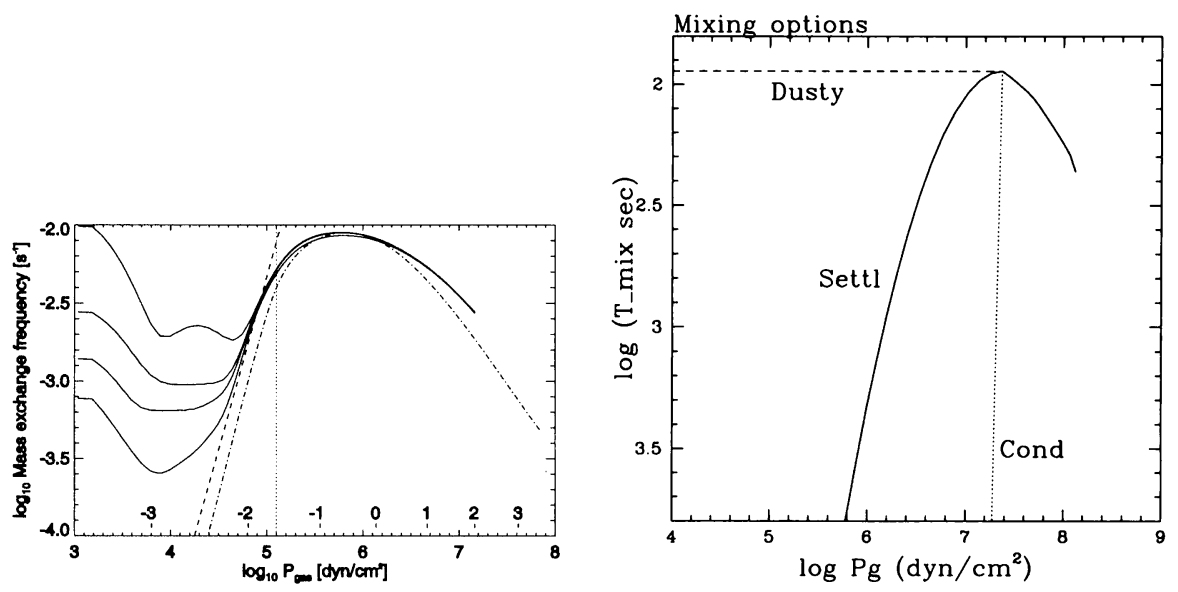

Figure 2. Left: Mass-exchange frequency (i.e. inverse mixing time scale) as a function of pressure from a hydrodynamical M-dwarf model atmosphere. The solid curves from top to bottom illustrate the effect of a successive removal of acoustic waves. The dashed line is an extrapolation towards a complete removal of acoustic contributions, the dashed-dotted line depicts an estimate from approximate linear theory. For details see text and Ludwig, Allard, \& Hauschildt (2002). Right: Mixing timescale as a function of pressure as used in our AMES-Settl models the $1900 \mathrm{~K}, \log g=5.5$ case (full line), compared to solutions available in settling code to mimick the dust-free or Cond case (dotted line) and the full dust or Dusty case (dashed line) models (Allard et al. 2001). The scales of the two plots have been chosen to span the same time and pressure range and allow a better comparison of the curves.

to the computed mass-exchange rate in Figure 2a. An advantage of this model is that our timescale prescription automatically scales with MLT changes as a function of $\mathrm{T}_{\text {eff }}$ or gravity.

\section{Results}

Figure 3a displays the thermal structures of the AMES-Settl models computed as described above. The cloud layers indicated by the thicker lines are now confined to within two pressure scale height(s) from the cloud base. While the main convection zone receeds with decreasing $\mathrm{T}_{\text {eff }}$, a detached convection zone is created within the cloud layer for the coolest models, as a result of the steep local dust opacity increase. The cloud layer encompasses the water vapor forming region around optical depths of $10^{-2}$ down to $T_{\text {eff }}=1700 \mathrm{~K}$. Below that temperature, the cloud sits too low in the atmosphere to warm up the near-IR spectrum, $\mathrm{CH}_{4}$ wins over $\mathrm{CO}$ in the chemistry, and methane bands appear in the spectrum near $3.5 \mu \mathrm{m}$. The strongest optical features (e.g. K I doublet at $\lambda 7666,7699 \AA$ ) which form around an optical depth of $10^{-4}$ however, always form outside the clouds across this $\mathrm{T}_{\text {eff }}$ range. For models with $\mathrm{T}_{\text {eff }} \leq 1100 K$, 

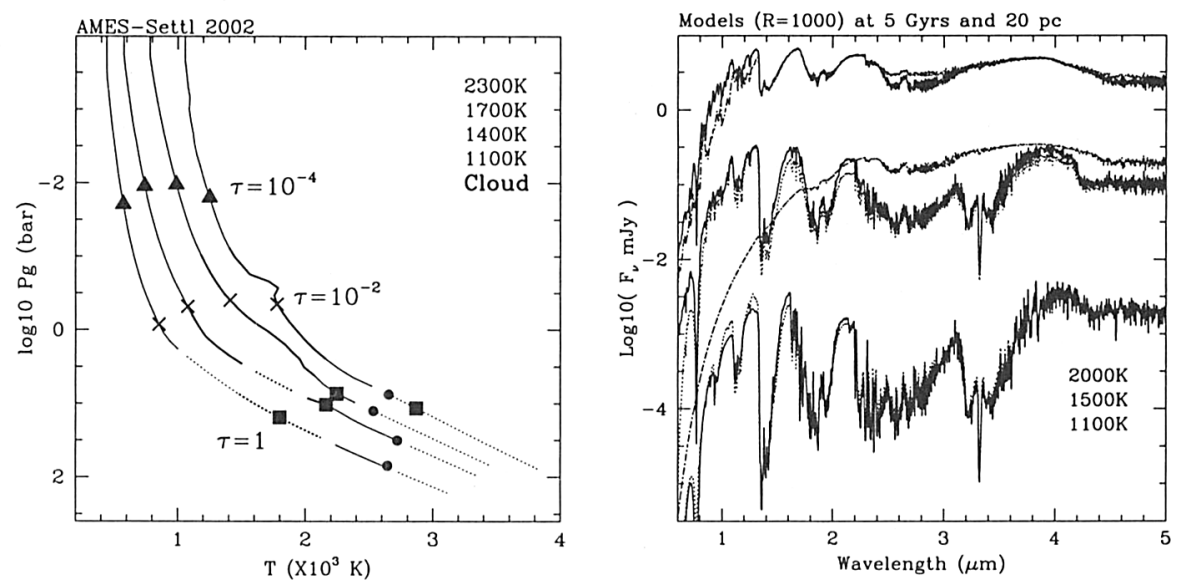

Figure 3. Left: Thermal structures of AMES-Settl models ranging from $\mathrm{T}_{\text {eff }}=2300$ to $1100 \mathrm{~K}$. Convective zones (dotted lines) as well are the onset of the innermost convection zone (circles) are indicated. The location of layers with optical depth at $1.2 \mu \mathrm{m}$ of $10^{-4}, 10^{-2}, 1.0$ are also shown, indicating the depth of formation of the strongest optical spectral features (triangles), of near-IR water vapor bands (crosses), and the continuum (squares). The cloud layer is shown by a thicker line. Right: Comparison of the spectral energy distributions of $\mathrm{T}_{\text {eff }}=2000$, 1500 , and $1100 \mathrm{~K}$ models in the 1) AMES-Settl approach (full line); 2) Cond limiting case (dotted line); and 3) Dusty limiting case (dashed line). The spectra have been flux calibrated based on the radii of Chabrier et al (2000) and their resolution reduced to allow the overal comparison.

the cloud layer is already forming below the entire spectral forming region (i.e. $\tau=1.0$ ), and corresponding spectra are essentially dust-free.

Figure $3 \mathrm{~b}$ shows how the AMES-Settl spectra differ from the corresponding Cond and Dusty models by Allard et al. (2001). On this plot one can observe that the effect of the presence of dust in the atmosphere is a progressive veiling of the optical to near-IR spectral distribution (with a corresponding IR flux redistribution) as $\mathrm{T}_{\text {eff }}$ decreases. The strength of this veiling increases with the radial cloud occupation as well as with decreasing $T_{\text {eff. }}$. In Dusty models, the radial occupation was constant and the veiling only becomes more important with decreasing $T_{\text {eff }}$, while no veiling is present in Cond models. In the AMESSettl models, we find a veiling as a function of $T_{\text {eff }}$ which is intermediate to Dusty and Cond models. At 2000K (topmost spectrum in Figure 3b), which corresponds to the M-to- $\mathrm{L}$ dwarf range where brown dwarfs have red $J-K$ colors, the AMES-Settl spectrum is brighter in the optical to red spectral range than its Dusty equivalent. But it is as bright as the Dusty models in the trough of the water bands at $H, K$ and $M$. At $1500 \mathrm{~K}$ (middle spectrum), i.e. in the late-L range where brown dwarfs have intermediate $J-K$ colors, the AMESSettl model is far fainter than Dusty and as bright as Cond models in the optical 
to red spectral range. But it carries more flux in the IR region than Cond models because of the dust layer. And below 1100K (lowermost spectrum), the AMESSettl is practically identical to the corresponding Cond model as the cloud layer is now well below the spectrum forming region of the atmosphere.

To appreciate the new models over a large $\mathrm{T}_{\text {eff }}$ range, we have interpolated the AMES-Settl model grid over the Chabrier et al. (2000) Dusty isochrones $\left(\mathrm{T}_{\text {eff }}-\log (g)\right.$ relation). Since the $\mathrm{T}_{\text {eff }}-\log (g)$ relation does not change significantly from Cond to Dusty, we consider this manipulation adequate. Figure 4 shows the result for 1 Gyr Dusty, Cond and AMES-Settl isochrones compared to a sample of brown dwarfs from the compilation by Leggett et al. (2002), Dahn et al. (2002), and Els et al. (2001) in the Mauna Kea MK photometric system (see Leggett et al. 2002 for details on the filter system).

\section{Discussion and Conclusions}

We have computed a new grid of model atmospheres including depletion of refractory elements (so called rain-out) in proportion to the sedimentation of grains, where the sedimentation has been opposed to condensation and convective updraft of condensable gas, and where the efficiency of the updraft mixing as a function of height in the atmosphere has been based upon the results of $3 \mathrm{D}$ radiation hydrodynamical convection studies. We find that the new models successfully reproduce globally the turn to the blue of $J-K$ colors that brown dwarfs from $\mathrm{L}$ to $\mathrm{T}$ types describe in color-magnitude diagrams. We therefore conclude that the adopted mixing timescale function is adequate and reflects the convective velocity drop in the radiative atmosphere zone of brown dwarfs.

$\mathrm{FeH}$ band strength and $J$ brightness have been observed to increase with spectral type in early T-type brown dwarfs (Burgasser et al. 2002, Dahn et al. 2002). While at this point spectral types do not strickly correspond to $T_{\text {eff }}$ changes (Stephens et al., elsewhere in this volume), this may be an indication (Burgasser et al. 2002) of a decrease of cloud surface coverage with decreasing $\mathrm{T}_{\text {eff. }}$ This is indeed an interesting idea. While the AMES-Settl models succeed in reproducing some brown dwarfs photometric features, detailed confrontation with observations will tell if this is the case for all features, and if indeed clear signs of cloud surface coverage variation are obtained.

The AMES-Settl models predict that grains in the atmosphere have sizes of the order $3 \mu \mathrm{m}$ or smaller. Growth to larger sizes is inhibited by two processes: (i) at high altitudes, sedimentation timescales are small and large grains cannot be supported by the relatively weak turbulent diffusion; (ii) at low altitudes, the diffusion timescales are short enough to allow the transport of the grains to deeper levels where they evaporate. Thus, our results disagree with that of Ackerman \& Marley (2001) and Marley et al. (2002) who obtain much larger grains $(\approx 10-100 \mu \mathrm{m})$, mostly because these authors assumed that grains would always grow rapidly enough for sedimentation to equilibrate with turbulent diffusion. However, our approach is not yet based on a fully consistent treatment of particle diffusion: grains may indeed be allowed to grow larger than predicted by our local approximation. We also derive only a mean size of condensed particles at any given altitude whereas Ackerman \& Marley obtain a distribution of sizes, which is conceptually more satisfying. In the limit when condensation is 


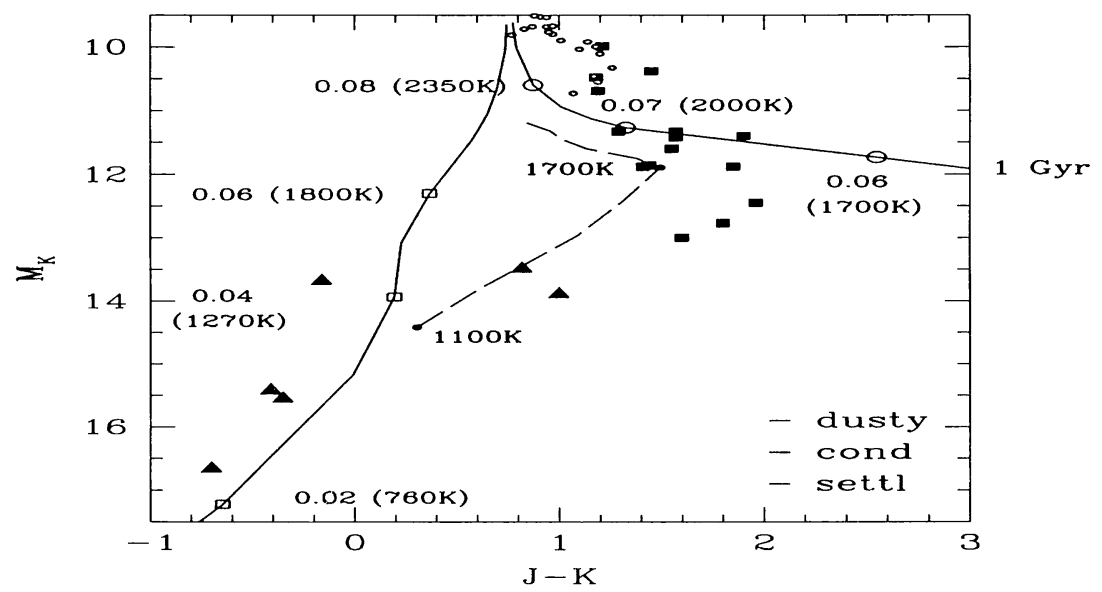

Figure 4. Comparison of the behavior of the AMES-Settl models (long-dashed line) to the Cond (full line to the left) and Dusty (full line to the right) limiting cases in the JK color plane. The Cond/Dusty 1 Gyr isochrones are taken from Chabrier et al. (2000) while the AMES-Settl colors are mapped onto the Dusty 1 Gyr $\mathrm{T}_{\text {eff-radii re- }}$ lation. The points represent low mass stars and brown dwarfs from the compilation by Leggett et al (2002), Dahn et al. (2002), and Els et al. (2001) in the Mauna Kea photometric system.

fast over the other timescales, their approach is certainly justified. However, we find that in most cases (and in particular in high-Teff brown dwarfs) it is not.

While our gravitational settling approach is self-consistent and free of adjustable parameters, it is simplified: we only solve the diffusion equation in a local approximation while, in the convection zone or in convective plumes, this process can reach global dimensions and would favor the levitation of larger grains than found in our current models. We also have neglected the effects of supersaturation which could allow the cloud base to sit somewhat deeper in the atmosphere. Finally, we must rely on the applicability of the Rossow (1978) timescales to brown dwarf atmosphere conditions. Helling et al. (2001) have explored the effects of compression and expansion of the plasma, due to convectively driven turbulence, on dust formation in the atmosphere of a $1900 \mathrm{~K}$ brown dwarf. They find that this process could induce formation of more dust grains of smaller and larger sizes than predicted in our AMES-Settl models. However the amplitude of the velocity disturbances as estimated from the typical MLT needed to produce these effects are no less than a factor 100 larger than prevailing in brown dwarfs atmospheres. Though the acoustic expansion waves may grow towards the outer atmosphere as hydrodynamical models have shown for solar and M dwarfs (LAH02, and references therein), this occurs only in the outermost atmosphere were dust has certainly rained out over time, leaving a metal-depleted plasma incapable of producing more dust even if stretched. Nevertheless, a full comprehension of the dust formation mechanisms at play in brown dwarfs will, in the end, come from other similar work on time-dependent grain growth and nucleation. 
With our static atmospheric studies, we provide some guidance, based upon observational constraints. We hope that with this work we have brought one more piece to the puzzle. The AMES-Settl thermal structures, synthetic spectra, and colors can be obtained from our anonymous ftp site (ftp://ftp.enslyon.fr/pub/users/CRAL/fallard/AMES-Settl-2002).

\section{References}

Ackerman, A. S., \& Marley, M. S. 2001, ApJ, 556, 872

Allard, F., Hauschildt, P. H., Alexander, D. R., Tamanai, A., \& Schweitzer, A. 2001, ApJ, 556, 357

Burgasser, A. J., Marley, M. S., Ackerman, A. S., Saumon, D., Lodders, K., Dahn, C. C., Harris, H. C., \& Kirkpatrick, J. D. 2002, ApJ, 571, L151

Chabrier, G., Baraffe, I., Allard, F., \& Hauschildt, P.H. 2000, ApJ, 542, 464

Dahn, C. C., Harris, H. C., Vrba, F. J., Guetter, H. H., Canzian, B., Henden, A. A., Levine, S. E., Luginbuhl, C. B., Monet, A. K. B., Monet, D. G., Pier, J. R., Stone, R. C., Walker, R. L., Burgasser, A. J., Gizis, J. E., Kirkpatrick, J. D., Liebert, J., \& Reid, I. N. 2002, AJ, in press

Els, S. G., Sterzik, M. F., Marchis, F., Pantin, E., Endl, M., \& Kürster, M. 2001, A\&A, 370, L1

Helling, C., Oevermann, M., Lüttke, M. J. H., Klein, R., \& Sedlmayr, E. 2001, A\&A, 376, 194

Leggett, S. K., Allard, F., Dahn, C., Hauschildt, P. H., Kerr, T. H., \& Rayner, J. 2000, ApJ, 535, 965

Leggett, S. K., Golimowski, D. A., Fan, X., Geballe, T. R., Knapp, G. R., Brinkmann, J., Csabai, I., Gunn, J. E., Hawley, S. L., Henry, T. J., Hindsley, R., Ivezić, Ž., Lupton, R. H., Pier, J. R., Schneider, D. P., Smith, J. A., Strauss, M. A., Uomoto, A., \& York, D. G. 2002, ApJ, 564, 452

Ludwig, H.-G., Allard, F., \& Hauschildt, P. H. 2002, A\&A, in press

Marley, M. S., Seager, S., Saumon, D., Lodders, K., Ackerman, A. S., Freedman, R. S., Fan, X., ApJ, 568, 335

Rossow, W. B. 1978, Icarus, 36, 1

Schweitzer, A., Gizis, J. E., Hauschildt, P. H., Allard, F., \& Reid, I. N. 2001, ApJ, 555, 368

Tsuji, T., Ohnaka, K., Aoki, W., \& Nakajima, T. 1996, ApJ, A\&A, 308, 29 